\title{
RF ACTIVITIES ON CAVITIES AND TURN KEY ACCELERATOR SYSTEMS AT ACCEL
}

\author{
M. Peiniger, M. Pekeler, C. Piel, P. vom Stein, H. Vogel, \\ ACCEL Instruments, Bergisch Gladbach, Germany
}

\begin{abstract}
Beside the pure manufacture of normal and superconducting cavities ACCEL Instruments has continuously expanded its RF activities for worldwide customers in research and industry. Our scope includes feasibility studies on special and large scale cavity applications, review and optimisation especially on SRF modules as well as the supply of complete accelerator linacs and systems including control systems and $\mathrm{rf}$ supply. These projects are performed in tight co operation with the worldwide leading experts, universities, and research institutes, respectively. Besides various other activities, which will be described in more detail, the most recent test results of the superconducting TESLA Cavities will be presented. Further the production of the PEP II style cavities $(476 \mathrm{MHz})$ will be described as well as the status of the fabrication of two $53 \mathrm{MHz}$ copper cavities for BNL's National Synchrotron Light Source. The recent project of the $500 \mathrm{MHz}$ Booster RF system for the Canadian Light Source will be introduced
\end{abstract}

\section{SUPERCONDUCTING CAVITIES}

A number of projects have been realised since the last two years. A few of them will be presented in more detail below.

\subsection{TESLA Cavities}

A series of 24 TESLA cavities (1.3 GHz, 9 cell cavities) have been fabricated and delivered to DESY. Based on previous test results the manufacturing parameters have been optimised.

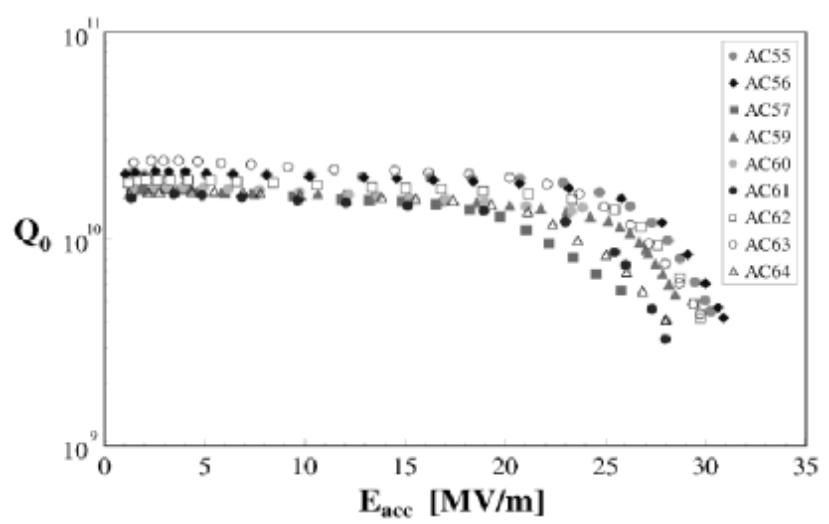

Figure 1: Performance of TESLA Cavities made by ACCEL
The test results after chemical preparation and high pressure water rinsing at DESY are shown in figure 1.

All cavities tested so far show the safe achievement of the desired parameters of $E_{\text {acc }}>25 \mathrm{MV} / \mathrm{m}$ with a quality factor of above $5^{*} 10^{9}$. Based on these results the realisation of TESLA is feasible based on the industrially fabricated accelerating cavities[1].

\subsection{Superconducting Modules for Storage Rings}

The CORNELL University has developed a superconducting $500 \mathrm{MHz}$ accelerator module originally designed as a "work horse" for a B-factory. In the meantime four such modules are installed in the CORNELL $\mathrm{e}^{+} \mathrm{e}^{-}$-ring CESR and six more are under fabrication at ACCEL for CORNELL (luminosity upgrade), the Taiwan Light Source and the Canadian Light Source. Details are given in ref. [2]

\section{NORMALCONDUCTING CAVITIES}

\subsection{Status of the PEP-II Cavity Production}

SLAC has ordered a total number of 10 PEP-II style cavities. Based on the excellent co-operation with SLAC we could manage the transfer of all machining data for the complex milling operations on the cavity bodies to our machines. Further to the machining operations the electroforming of the cooling jacket is well completed. Figure 2 shows the cavity during the electroforming process. The next milestone will be the electron beam welding of the coupling ports to the cavity bodies.

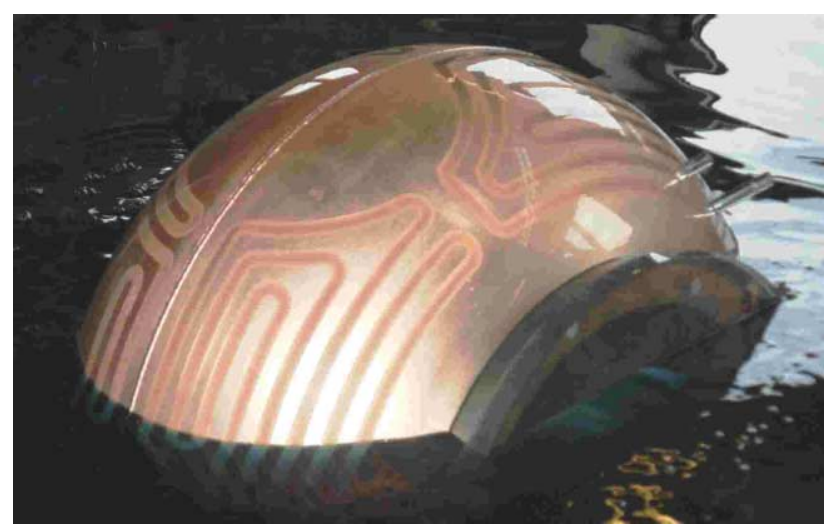

Figure 2: PEP-II style cavity during electroforming of the cooling jacket 


\section{$2.2 \mathrm{RF}$ System for the $500 \mathrm{MHz}$ Booster of the Canadian Light Source}

The booster synchrotron of the Canadian Light Source CLS as well as ELETTRA will be equipped with 500 MHz Five Cell Cavities. The cavities are based on the DESY/PETRA design and will be delivered turn-key, including all necessary auxiliaries as high power coupler, tuners and signal pick-up loops. The basic parameters of the cavity are summarised in table 1 below.

Table 1: Basic Parameters for Booster Cavities

\begin{tabular}{|l|l|l|}
\hline Frequency & 500 & $\mathrm{MHz}$ \\
\hline $\mathrm{R}_{\mathrm{S}}=\mathrm{U}^{2} /\left(2 \mathrm{P}_{\text {in }}\right)$ & $>14.5$ & $\mathrm{M} \Omega$ \\
\hline $\mathrm{Q}_{0}$ & 29.000 & \\
\hline RF Power & $40 / 65$ & $\mathrm{KW}$ \\
\hline Field Flatness & $+/-5$ & $\%$ \\
\hline Beam tube flange & $\mathrm{DN} \mathrm{63/100}$ & $\mathrm{CF}$ \\
\hline Pick up connector & 4 & N-Type \\
\hline Cooling connector & 2 & in/out \\
\hline
\end{tabular}

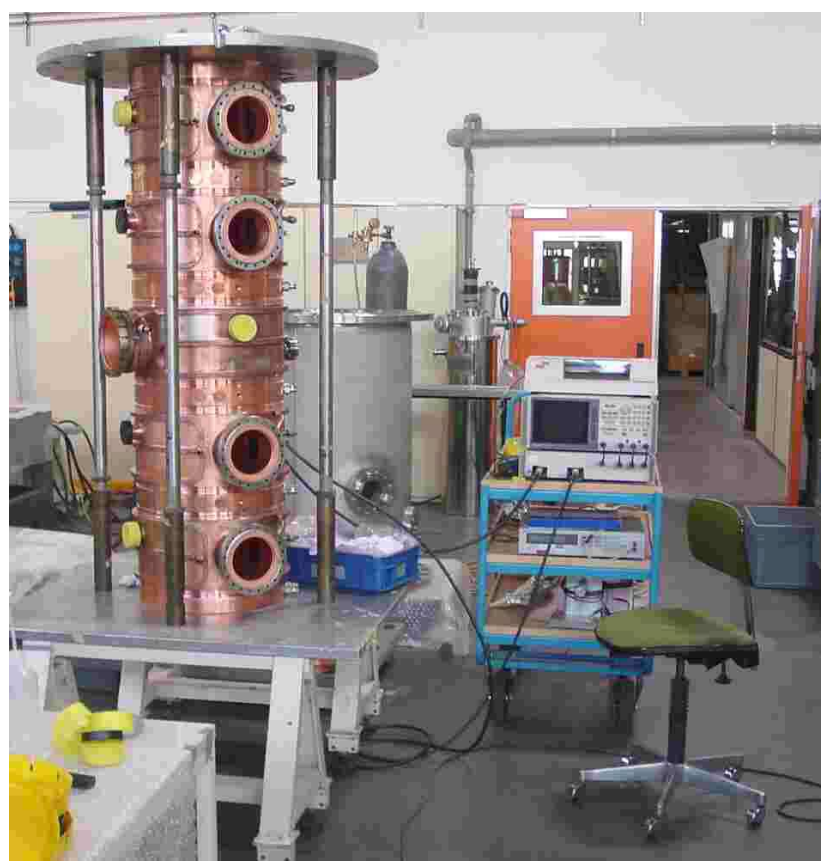

Figure 3: $500 \mathrm{MHz}$ cavity piled up for frequency and field profile tuning

The ELETTRA Booster cavity will be high power tested including the cavity components like tuners, power couplers, and field-pick ups. The scope of delivery for CLS includes also the low level rf control electronics (see figure 4).

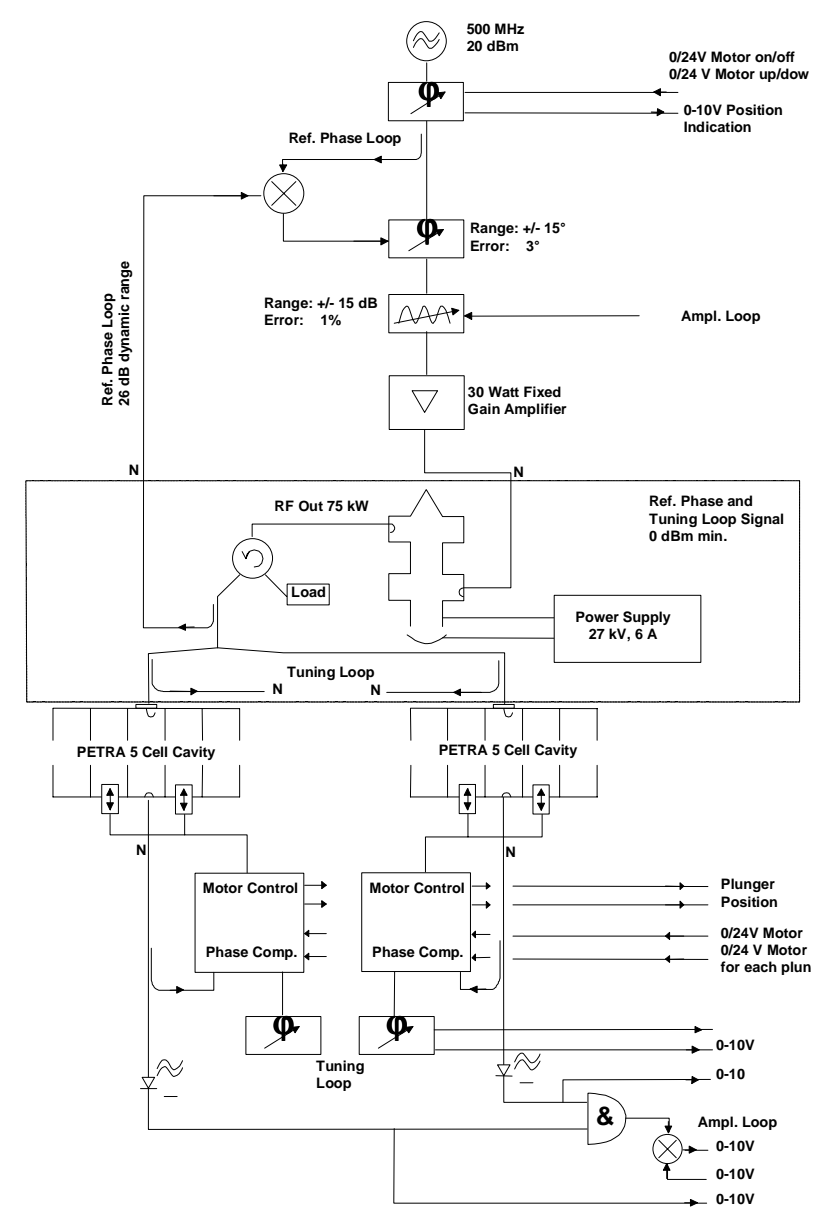

Figure 4: layout of the low level control system for the CLS booster RF system (high power amplifier excluded)

\subsection{Status of the BNL Cavity production}

For Brookhaven's National Synchrotron Light Source two cavities are under fabrication besides the two already delivered in August 1997. The machining of the cavity parts has almost been completed and the final Electron Beam welding operations and he RF tuning work is scheduled for June and July 2001.

\section{DESIGN AND FEASIBILITY STUDIES}

\subsection{Landau Module}

The installation of Landau Cavities in storage rings to improve beam life time limited by the Touchek effect is a recently discussed topic. BESSY has decided to approach this topic with a superconducting third harmonic cavity. Before start of the production of this accelerator module a design study was performed with a detailed look on the $\mathrm{RF}$ and the cryogenic aspects of such a module under the specific tight space limitations of an existing storage ring. The cavity design is a scaled CORNELL CESR-III cavity and it could be shown that the module fits into an overall space of $800 \mathrm{~mm}$ (flange to flange) with acceptable cryogenic performance (standby losses below 6 Watt) [3] 


\subsection{Superconducting CH-Structure}

Acceleration of heavy ions with $\mathrm{IH}$ - and $\mathrm{CH}$ - type room temperature structures is commonly used. Based on a new concept of using superconducting $\mathrm{CH}$-mode structures a design study is under preparation for such cavites with focus on :

- Optimisation of cavity layout with respect to preparation technology (chemistry, high pressure water rinsing)

- Optimisation of cavity layout with respect to fabrication technologies (metal sheet forming, welding, brazing...)

\section{CO-OPERATIONS}

We appreciate the open and very successful co operation with Universities and Research Institutes in the field of accelerator and magnet technology which may be illustrated by the following examples.

Based on a detailed technology exchange with DESY we mastered the manufacturing technology for S-Band accelerating cavities and applied this with great success for the $100 \mathrm{MeV}$ injector for the Swiss Light Source.
Technology Transfer Agreements with CERN allow us to perform cryogenic cold test for development of new accelerator modules

The co-operation with CORNELL enables us to deliver superconducting accelerator modules with guaranteed performance

Test installations at the University of Dortmund have been used for development and test of S-Band accelerating components and electron sources for the SLS injector linac.

\section{REFERENCES}

[1] TESLA The Superconducting Electron-Positron Linear Collider with an Integrated X-Ray Laser Laboratory, Technical Design Report Vol. II, DESY 2001-011, ECFA 2001-209, TESLA Report 2001-23, TESLA FEL 2001-05, March 2001.

[2] M. Pekeler et al, "Industrial Production of Turn Key Superconducting Accelerator Modules for High Current Storage Rings", ACCEL Instruments GmbH, Proceedings this conference

[3] P. vom Stein et al, "A Superconducting Landau Accelerator Module for BESSY II", ACCEL Instruments $\mathrm{GmbH}$, Proceedings this conference 